SLAC-PUB-13998

\title{
AdS/QCD, Light-Front Holography, and the Nonperturbative Running Coupling
}

\author{
Stanley J. Brodsky, ${ }^{a}$ Guy F. de Téramond, ${ }^{b}$ and Alexandre Deur ${ }^{c}$ \\ ${ }^{a}$ SLAC National Accelerator Laboratory Stanford University, Stanford, CA 94309, USA \\ ${ }^{b}$ Universidad de Costa Rica, San José, Costa Rica \\ ${ }^{c}$ Thomas Jefferson National Accelerator Facility, Newport News, VA 23606, USA
}

\begin{abstract}
The combination of Anti-de Sitter space (AdS) methods with light-front (LF) holography provides a remarkably accurate first approximation for the spectra and wavefunctions of meson and baryon light-quark bound states. The resulting bound-state Hamiltonian equation of motion in QCD leads to relativistic light-front wave equations in terms of an invariant impact variable $\zeta$ which measures the separation of the quark and gluonic constituents within the hadron at equal light-front time. These equations of motion in physical space-time are equivalent to the equations of motion which describe the propagation of spin- $J$ modes in anti-de Sitter (AdS) space. The eigenvalues give the hadronic spectrum, and the eigenmodes represent the probability distributions of the hadronic constituents at a given scale. A positive-sign confining dilaton background modifying AdS space gives a very good account of meson and baryon spectroscopy and form factors. The light-front holographic mapping of this model also leads to a non-perturbative effective coupling $\alpha_{s}^{A d S}\left(Q^{2}\right)$ which agrees with the effective charge defined by the Bjorken sum rule and lattice simulations. It displays a transition from perturbative to nonperturbative conformal regimes at a momentum scale $\sim 1 \mathrm{GeV}$. The resulting $\beta$-function appears to capture the essential characteristics of the full $\beta$-function of QCD, thus giving further support to the application of the gauge/gravity duality to the confining dynamics of strongly coupled QCD.
\end{abstract}




\section{Introduction}

The AdS/CFT correspondence [1] between a gravity or string theory on a higher dimensional Anti-de Sitter (AdS) space-time with conformal gauge field theories (CFT) in physical space-time has brought a new set of tools for studying the dynamics of strongly coupled quantum field theories, and it has led to new analytical insights into the confining dynamics of QCD. The AdS/CFT duality provides a gravity description in a $(d+1)$-dimensional AdS space-time in terms of a flat $d$-dimensional conformallyinvariant quantum field theory defined at the AdS asymptotic boundary. [2] Thus, in principle, one can compute physical observables in a strongly coupled gauge theory in terms of a classical gravity theory. Since the quantum field theory dual to $\mathrm{AdS}_{5} \mathrm{space}$ in the original correspondence [1] is conformal, the strong coupling of the dual gauge theory is constant, and its $\beta$-function is zero. Thus one must consider a deformed AdS space in order to simulate color confinement and have a running coupling $\alpha_{s}^{A d S}\left(Q^{2}\right)$ for the gauge theory side of the correspondence. As we shall review here, a positive-sign confining dilaton background modifying AdS space gives a very good account of meson and baryon spectroscopy and their elastic form factors. The light-front holographic mapping of this model also leads to a non-perturbative effective coupling $\alpha_{s}^{A d S}\left(Q^{2}\right)$ which agrees with the effective charge defined by the Bjorken sum rule and lattice simulations 3 .

In the standard applications of AdS/CFT methods, one begins with Maldacena's duality between the conformal supersymmetric $S O(4,2)$ gauge theory and a semiclassical supergravity string theory defined in a 10 dimension $\operatorname{AdS}_{5} \times S^{5}$ space-time. [1] The essential mathematical tool underlying Maldacena's observation is the fact that the effects of scale transformations in a conformal theory can be mapped to the $z$ dependence of amplitudes in $\mathrm{AdS}_{5}$ space. QCD is not conformal but there is in fact much empirical evidence from lattice, Dyson Schwinger theory and effective charges that the QCD $\beta$ function vanishes in the infrared. 4] The QCD infrared fixed point arises since the propagators of the confined quarks and gluons in the loop integrals contributing to the $\beta$ function have a maximal wavelength. [5] The decoupling of quantum loops in the infrared is analogous to QED where vacuum polarization corrections to the photon propagator decouple at $Q^{2} \rightarrow 0$.

We thus begin with a conformal approximation to QCD to model an effective dual gravity description in $\mathrm{AdS}$ space. One uses the five-dimensional $\mathrm{AdS}_{5}$ geometrical representation of the conformal group to represent scale transformations within the conformal window. Confinement can be introduced with a sharp cut-off in the infrared region of AdS space, as in the "hard-wall" model, [6] or, more successfully, using a dilaton background in the fifth dimension to produce a smooth cutoff at large distances as in the "soft-wall" model. [7] The soft-wall AdS/CFT model with a positive-sign dilaton-modified AdS space leads to the potential $U(z)=\kappa^{4} z^{2}+2 \kappa^{2}(L+S-1)$, [8] in the fifth dimension coordinate $z$. We assume a dilaton profile $\exp \left(+\kappa^{2} z^{2}\right)$ [8, 9, 10, 11, with opposite sign to that of Ref. [7]. The resulting spectrum reproduces linear Regge 
trajectories, where $\mathcal{M}^{2}(S, L, n)$ is proportional to the internal spin, orbital angular momentum $L$ and the principal quantum number $n$.

The modified metric induced by the dilaton can be interpreted in AdS space as a gravitational potential for an object of mass $m$ in the fifth dimension: $V(z)=$ $m c^{2} \sqrt{g_{00}}=m c^{2} R e^{ \pm \kappa^{2} z^{2} / 2} / z$. In the case of the negative solution, the potential decreases monotonically, and thus an object in AdS will fall to infinitely large values of $z$. For the positive solution, the potential is non-monotonic and has an absolute minimum at $z_{0}=1 / \kappa$. Furthermore, for large values of $z$ the gravitational potential increases exponentially, confining any object to distances $\langle z\rangle \sim 1 / \kappa$. [8] We thus will choose the positive sign dilaton solution. This additional warp factor leads to a well-defined scale-dependent effective coupling. Introducing a positive-sign dilaton background is also relevant for describing chiral symmetry breaking, [10] since the expectation value of the scalar field associated with the quark mass and condensate does not blow-up in the far infrared region of AdS, in contrast with the original model. [7]

Glazek and Schaden [12] have shown that a harmonic oscillator confining potential naturally arises as an effective potential between heavy quark states when one stochastically eliminates higher gluonic Fock states. Also, Hoyer [13] has argued that the Coulomb and a linear potentials are uniquely allowed in the Dirac equation at the classical level. The linear potential becomes a harmonic oscillator potential in the corresponding Klein-Gordon equation.

Light-front (LF) quantization is the ideal framework for describing the structure of hadrons in terms of their quark and gluon degrees of freedom. The light-front wavefunctions (LFWFs) of bound states in QCD are relativistic generalizations of the Schrödinger wavefunctions, but they are determined at fixed light-front time $\tau=x^{+}=$ $x^{0}+x^{3}$, the time marked by the front of a light wave [14], rather than at fixed ordinary time $t$. They play the same role in hadron physics that Schrödinger wavefunctions play in atomic physics. [15] The simple structure of the LF vacuum provides an unambiguous definition of the partonic content of a hadron in QCD.

Light-front holography [19, 16, 17, 18, 8, 20] connects the equations of motion in AdS space and the Hamiltonian formulation of QCD in physical space-time quantized on the light front at fixed LF time. This correspondence provides a direct connection between the hadronic amplitudes $\Phi(z)$ in AdS space with LF wavefunctions $\phi(\zeta)$ describing the quark and gluon constituent structure of hadrons in physical space-time. In the case of a meson, $\zeta=\sqrt{x(1-x) \mathbf{b}_{\perp}^{2}}$ is a Lorentz invariant coordinate which measures the distance between the quark and antiquark; it is analogous to the radial coordinate $r$ in the Schrödinger equation. In effect $\zeta$ represents the off-light-front energy shell or invariant mass dependence of the bound state; it allows the separation of the dynamics of quark and gluon binding from the kinematics of constituent spin and internal orbital angular momentum. [19] Light-front holography thus provides a connection between the description of hadronic modes in AdS space and the Hamiltonian formulation of QCD in physical space-time quantized on the light-front at fixed LF time $\tau$.

The mapping between the LF invariant variable $\zeta$ and the fifth-dimension AdS 
coordinate $z$ was originally obtained by matching the expression for electromagnetic current matrix elements in AdS space [21] with the corresponding expression for the current matrix element, using LF theory in physical space time. [16] It has also been shown that one obtains the identical holographic mapping using the matrix elements of the energy-momentum tensor, [18, 22] thus verifying the consistency of the holographic mapping from AdS to physical observables defined on the light front.

The resulting equation for the mesonic $q \bar{q}$ bound states at fixed light-front time has the form of a single-variable relativistic Lorentz invariant Schrödinger equation

$$
\left(-\frac{d^{2}}{d \zeta^{2}}-\frac{1-4 L^{2}}{4 \zeta^{2}}+U(\zeta)\right) \phi(\zeta)=\mathcal{M}^{2} \phi(\zeta)
$$

where the confining potential is $U(\zeta)=\kappa^{4} \zeta^{2}+2 \kappa^{2}(L+S-1)$ in the soft-wall model. Its eigenvalues determine the hadronic spectra and its eigenfunctions are related to the light-front wavefunctions of hadrons for general spin and orbital angular momentum. This LF wave equation serves as a semiclassical first approximation to QCD, and it is equivalent to the equations of motion which describe the propagation of spin- $J$ modes in AdS space. The resulting light-front wavefunctions provide a fundamental description of the structure and internal dynamics of hadronic states in terms of their constituent quark and gluons. There is only one parameter, the mass scale $\kappa \sim 1 / 2 \mathrm{GeV}$, which enters the confinement potential. In the case of mesons $S=0,1$ is the combined spin of the $q$ and $\bar{q}, L$ is their relative orbital angular momentum as determined by the hadronic light-front wavefunctions.

The concept of a running coupling $\alpha_{s}\left(Q^{2}\right)$ in QCD is usually restricted to the perturbative domain. However, as in QED, it is useful to define the coupling as an analytic function valid over the full space-like and time-like domains. The study of the non-Abelian QCD coupling at small momentum transfer is a complex problem because of gluonic self-coupling and color confinement. We will show that the lightfront holographic mapping of classical gravity in AdS space, modified by a positive-sign dilaton background, leads to a non-perturbative effective coupling $\alpha_{s}^{A d S}\left(Q^{2}\right)$ which is in agreement with hadron physics data extracted from different observables, as well as with the predictions of models with built-in confinement and lattice simulations.

\section{The Hadron Spectrum and Form Factors in Light- Front AdS/QCD}

The meson spectrum predicted by Eq. 1 has a string-theory Regge form $\mathcal{M}^{2}=4 \kappa^{2}(n+$ $L+S / 2)$; i.e., the square of the eigenmasses are linear in both $L$ and $n$, where $n$ counts the number of nodes of the wavefunction in the radial variable $\zeta$. This is illustrated for the pseudoscalar and vector meson spectra in Fig. 1, where the data are from Ref. [23]. The pion $(S=0, n=0, L=0)$ is massless for zero quark mass, consistent with the chiral invariance of massless QCD. Thus one can compute the hadron spectrum by 
simply adding $4 \kappa^{2}$ for a unit change in the radial quantum number, $4 \kappa^{2}$ for a change in one unit in the orbital quantum number $L$ and $2 \kappa^{2}$ for a change of one unit of spin $S$. Remarkably, the same rule holds for three-quark baryons as we shall show below.
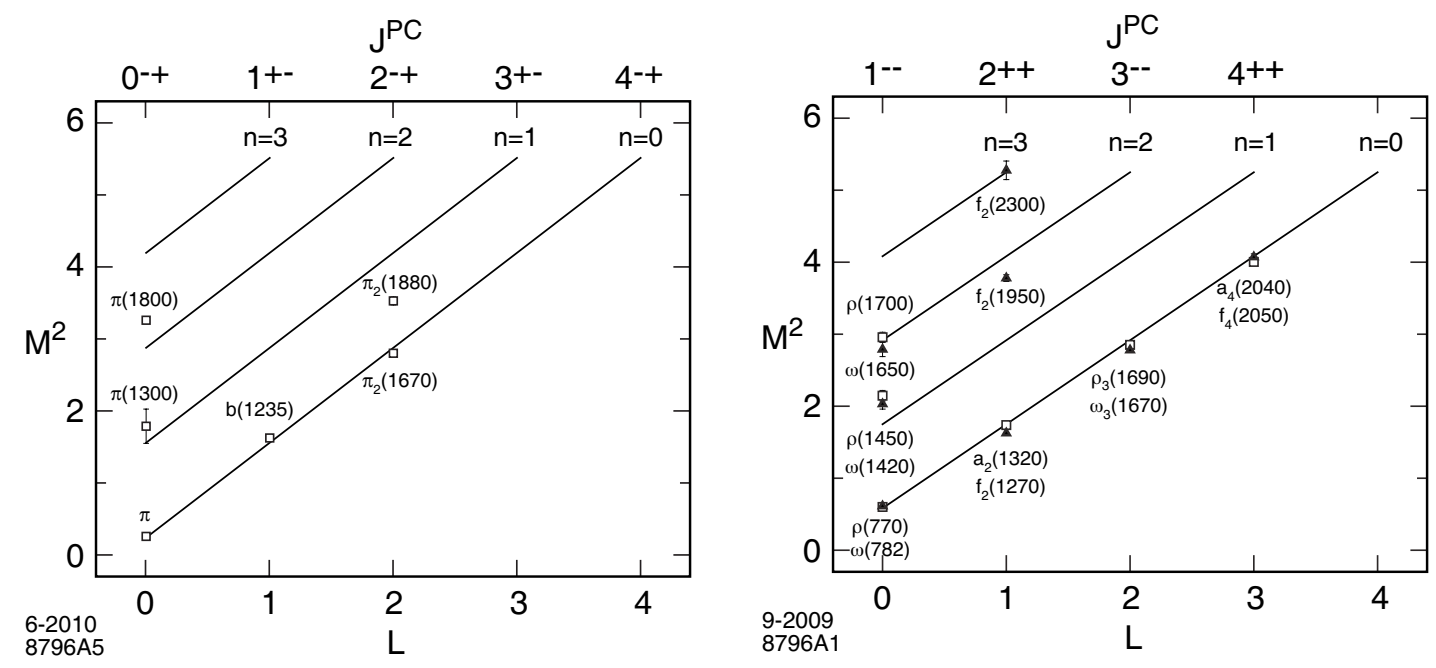

Figure 1: Parent and daughter Regge trajectories for (a) the $\pi$-meson family with $\kappa=0.6 \mathrm{GeV}$; and (b) the $I=1 \rho$-meson and $I=0 \omega$-meson families with $\kappa=0.54 \mathrm{GeV}$

In the light-front formalism, one sets boundary conditions at fixed $\tau$ and then evolves the system using the light-front (LF) Hamiltonian $P^{-}=P^{0}-P^{3}=i d / d \tau$. The invariant Hamiltonian $H_{L F}=P^{+} P^{-}-P_{\perp}^{2}$ then has eigenvalues $\mathcal{M}^{2}$ where $\mathcal{M}$ is the physical mass. Its eigenfunctions are the light-front eigenstates whose Fock state projections define the frame-independent light-front wavefunctions. The eigensolutions of Eq. 1 provide the light-front wavefunctions of the valence Fock state of the hadrons $\psi\left(x, \mathbf{b}_{\perp}\right)$ as illustrated for the pion in Fig. 2 for the soft (a) and hard wall (b) models. The resulting distribution amplitude has a broad form $\phi_{\pi}(x) \sim \sqrt{x(1-x)}$ which is compatible with moments determined from lattice gauge theory. One can then immediately compute observables such as hadronic form factors (overlaps of LFWFs), structure functions (squares of LFWFs), as well as the generalized parton distributions and distribution amplitudes which underly hard exclusive reactions. For example, hadronic form factors can be predicted from the overlap of LFWFs in the Drell-Yan West formula. The prediction for the space-like pion form factor is shown in Fig. 2(c). The pion form factor and the vector meson poles residing in the dressed current in the soft wall model require choosing a value of $\kappa$ smaller by a factor of $1 / \sqrt{2}$ than the canonical value of $\kappa$ which determines the mass scale of the hadronic spectra. This shift is apparently due to the fact that the transverse current in $e^{+} e^{-} \rightarrow q \bar{q}$ creates a quark pair with $L^{z}= \pm 1$ instead of the $L^{z}=0 q \bar{q}$ composition of the vector mesons in the spectrum.

Individual hadrons in AdS/QCD are identified by matching the power behavior of 

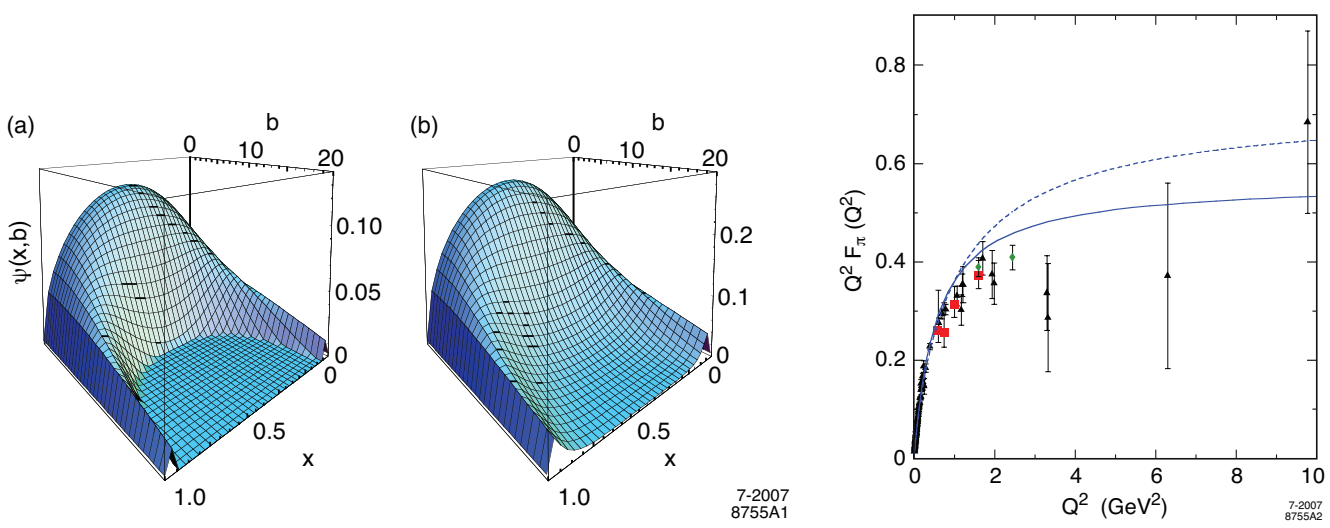

Figure 2: Pion LF wavefunction $\psi_{\pi}\left(x, \mathbf{b}_{\perp}\right)$ for the AdS/QCD (a) hard-wall $\left(\Lambda_{Q C D}=\right.$ $0.32 \mathrm{GeV}$ ) and (b) soft-wall $(\kappa=0.375 \mathrm{GeV})$ models. (c) Space-like scaling behavior for $Q^{2} F_{\pi}\left(Q^{2}\right)$. The continuous line is the prediction of the soft-wall model for $\kappa=0.375$ $\mathrm{GeV}$. The dashed line is the prediction of the hard-wall model for $\Lambda_{\mathrm{QCD}}=0.22 \mathrm{GeV}$. The triangles are the data compilation of Baldini.

the hadronic amplitude at the AdS boundary at small $z$ to the twist $\tau$ of its interpolating operator at short distances $x^{2} \rightarrow 0$, as required by the AdS/CFT dictionary. The twist also equals the dimension of fields appearing in chiral super-multiplets; [24] thus the twist of a hadron equals the number of constituents plus the relative orbital angular momentum. One then can apply light-front holography to relate the amplitude eigensolutions in the fifth dimension coordinate $z$ to the LF wavefunctions in the physical space-time variable $\zeta$.

Equation (1) was derived by taking the LF bound-state Hamiltonian equation of motion as the starting point. [19] The term $L^{2} / \zeta^{2}$ in the LF equation of motion (1) is derived from the reduction of the LF kinetic energy when one transforms to twodimensional cylindrical coordinates $(\zeta, \varphi)$, in analogy to the $\ell(\ell+1) / r^{2}$ Casimir term in Schrödinger theory. One thus establishes the interpretation of $L$ in the AdS equations of motion. The interaction terms build confinement corresponding to the dilaton modification of AdS space [19]. The duality between these two methods provides a direct connection between the description of hadronic modes in AdS space and the Hamiltonian formulation of QCD in physical space-time quantized on the light-front at fixed LF time $\tau$.

The identification of orbital angular momentum of the constituents is a key element in the description of the internal structure of hadrons using holographic principles. In our approach quark and gluon degrees of freedom are explicitly introduced in the gauge/gravity correspondence, 25] in contrast with the usual AdS/QCD framework [26, 27] where axial and vector currents become the primary entities as in effective chiral theory. Unlike the top-down string theory approach, one is not limited to hadrons of maximum spin $J \leq 2$, and one can study baryons with finite color $N_{C}=3$. Higher 
spin modes follow from shifting dimensions in the AdS wave equations. In the soft-wall model the usual Regge behavior is found $\mathcal{M}^{2} \sim n+L$, predicting the same multiplicity of states for mesons and baryons as observed experimentally. 28] It is possible to extend the model to hadrons with heavy quark constituents by introducing nonzero quark masses and short-range Coulomb corrections. For other recent calculations of the hadronic spectrum based on AdS/QCD, see Refs. [29, 30, 31, 32, 33, 34, 35, 36, 37, 38, 39, 40]. Other recent computations of the pion form factor are given in Refs. [41, 42,

For baryons, the light-front wave equation is a linear equation determined by the LF transformation properties of spin $1 / 2$ states. A linear confining potential $U(\zeta) \sim \kappa^{2} \zeta$ in the LF Dirac equation leads to linear Regge trajectories. [43] For fermionic modes the light-front matrix Hamiltonian eigenvalue equation $D_{L F}|\psi\rangle=\mathcal{M}|\psi\rangle, H_{L F}=D_{L F}^{2}$, in a $2 \times 2$ spinor component representation is equivalent to the system of coupled linear equations

$$
\begin{aligned}
-\frac{d}{d \zeta} \psi_{-}-\frac{\nu+\frac{1}{2}}{\zeta} \psi_{-}-\kappa^{2} \zeta \psi_{-} & =\mathcal{M} \psi_{+}, \\
\frac{d}{d \zeta} \psi_{+}-\frac{\nu+\frac{1}{2}}{\zeta} \psi_{+}-\kappa^{2} \zeta \psi_{+} & =\mathcal{M} \psi_{-}
\end{aligned}
$$

with eigenfunctions

$$
\begin{aligned}
& \psi_{+}(\zeta) \sim z^{\frac{1}{2}+\nu} e^{-\kappa^{2} \zeta^{2} / 2} L_{n}^{\nu}\left(\kappa^{2} \zeta^{2}\right) \\
& \psi_{-}(\zeta) \sim z^{\frac{3}{2}+\nu} e^{-\kappa^{2} \zeta^{2} / 2} L_{n}^{\nu+1}\left(\kappa^{2} \zeta^{2}\right)
\end{aligned}
$$

and eigenvalues $\mathcal{M}^{2}=4 \kappa^{2}(n+\nu+1)$.

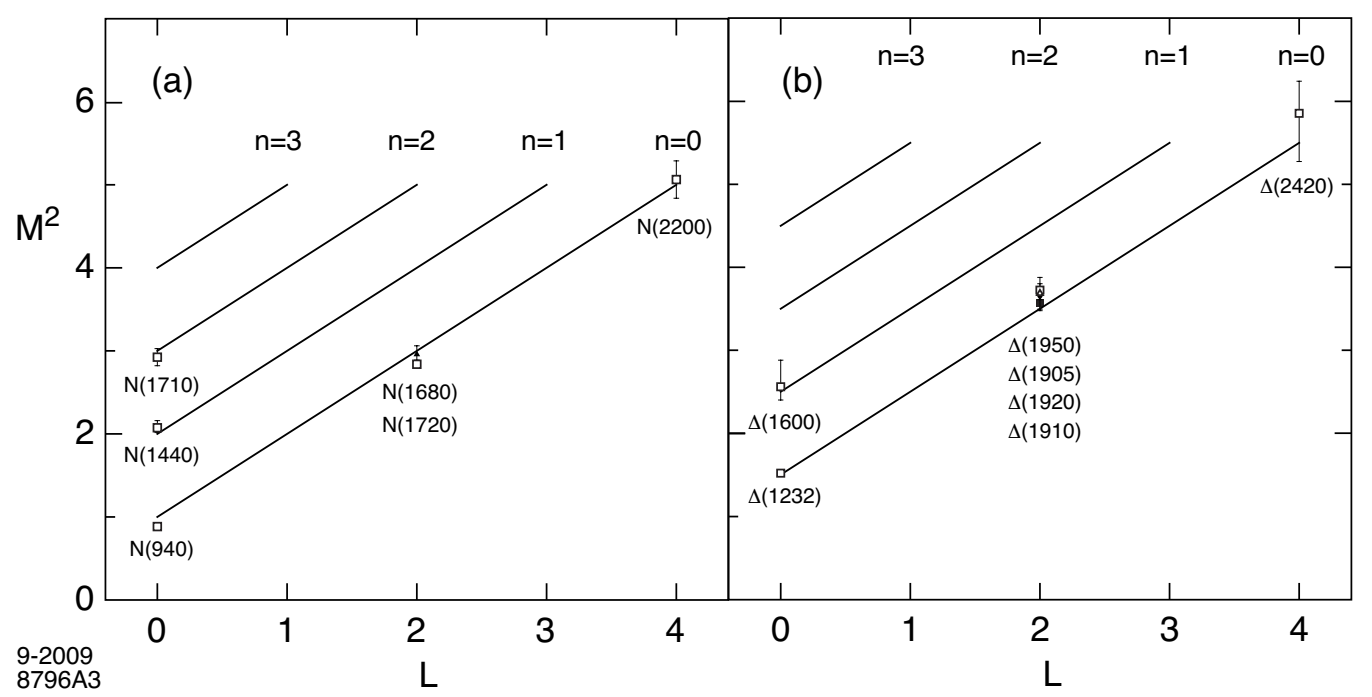

Figure 3: 56 Regge trajectories for the $N$ and $\Delta$ baryon families for $\kappa=0.5 \mathrm{GeV}$. 
The baryon interpolating operator $\mathcal{O}_{3+L}=\psi D_{\left\{\ell_{1}\right.} \ldots D_{\ell_{q}} \psi D_{\ell_{q+1}} \ldots D_{\left.\ell_{m}\right\}} \psi, L=$ $\sum_{i=1}^{m} \ell_{i}$, is a twist 3 , dimension $9 / 2+L$ with scaling behavior given by its twistdimension $3+L$. We thus require $\nu=L+1$ to match the short distance scaling behavior. Higher spin modes are obtained by shifting dimensions for the fields. Thus, as in the meson sector, the increase in the mass squared for higher baryonic state is $\Delta n=4 \kappa^{2}, \Delta L=4 \kappa^{2}$ and $\Delta S=2 \kappa^{2}$, relative to the lowest ground state, the proton. Since our starting point to find the bound state equation of motion for baryons is the light-front, we fix the overall energy scale identical for mesons and baryons by imposing chiral symmetry to the pion [20] in the LF Hamiltonian equations. By contrast, if we start with a five-dimensional action for a scalar field in presence of a positive sign dilaton, the pion is automatically massless.

The predictions for the 56-plet of light baryons under the $S U(6)$ flavor group are shown in Fig. 3. As for the predictions for mesons in Fig. 1, only confirmed PDG [23] states are shown. The Roper state $N(1440)$ and the $N(1710)$ are well accounted for in this model as the first and second radial states. Likewise the $\Delta(1660)$ corresponds to the first radial state of the $\Delta$ family. The model is successful in explaining the important parity degeneracy observed in the light baryon spectrum, such as the $L=2$, $N(1680)-N(1720)$ degenerate pair and the $L=2, \Delta(1905), \Delta(1910), \Delta(1920), \Delta(1950)$ states which are degenerate within error bars. Parity degeneracy of baryons is also a property of the hard wall model, but radial states are not well described in this model. [44]

As an example of the scaling behavior of a twist $\tau=3$ hadron, we compute the spin non-flip nucleon form factor in the soft wall model. [43] The proton and neutron Dirac form factors are given by

$$
\begin{gathered}
F_{1}^{p}\left(Q^{2}\right)=\int d \zeta J(Q, \zeta)\left|\psi_{+}(\zeta)\right|^{2}, \\
F_{1}^{n}\left(Q^{2}\right)=-\frac{1}{3} \int d \zeta J(Q, \zeta)\left[\left|\psi_{+}(\zeta)\right|^{2}-\left|\psi_{-}(\zeta)\right|^{2}\right],
\end{gathered}
$$

where $F_{1}^{p}(0)=1, \quad F_{1}^{n}(0)=0$. The non-normalizable mode $J(Q, z)$ is the solution of the AdS wave equation for the external electromagnetic current in presence of a dilaton background $\exp \left( \pm \kappa^{2} z^{2}\right)$. [17, 45] Plus and minus components of the twist 3 nucleon LFWF are

$$
\psi_{+}(\zeta)=\sqrt{2} \kappa^{2} \zeta^{3 / 2} e^{-\kappa^{2} \zeta^{2} / 2}, \quad \Psi_{-}(\zeta)=\kappa^{3} \zeta^{5 / 2} e^{-\kappa^{2} \zeta^{2} / 2} .
$$

The results for $Q^{4} F_{1}^{p}\left(Q^{2}\right)$ and $Q^{4} F_{1}^{n}\left(Q^{2}\right)$ and are shown in Fig. 4. [46]

\section{Nonperturbative Running Coupling from Light- Front Holography}

The definition of the running coupling in perturbative quantum field theory is schemedependent. As discussed by Grunberg, [47] an effective coupling or charge can be 

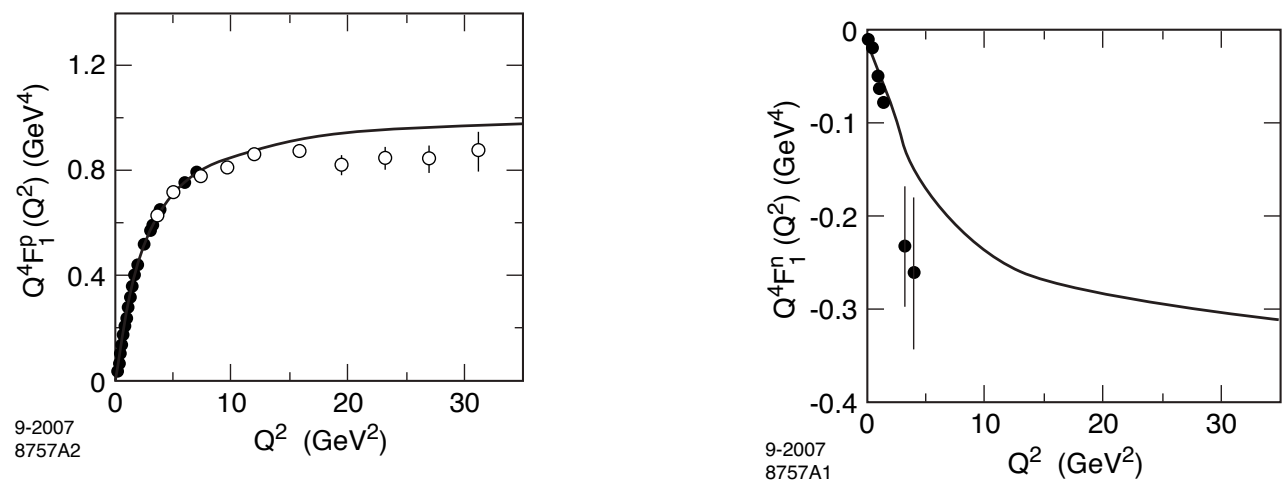

Figure 4: Predictions for $Q^{4} F_{1}^{p}\left(Q^{2}\right)$ and $Q^{4} F_{1}^{n}\left(Q^{2}\right)$ in the soft wall model for $\kappa=0.424$ $\mathrm{GeV}$.

defined directly from physical observables. Effective charges defined from different observables can be related to each other in the leading-twist domain using commensurate scale relations (CSR). [48. The potential between infinitely heavy quarks can be defined analytically in momentum transfer space as the product of the running coupling times the Born gluon propagator: $V(q)=-4 \pi C_{F} \alpha_{V}(q) / q^{2}$. This effective charge defines a renormalization scheme - the $\alpha_{V}$ scheme of Appelquist, Dine, and Muzinich. [49] In fact, the holographic coupling $\alpha_{s}^{A d S}\left(Q^{2}\right)$ can be considered to be the nonperturbative extension of the $\alpha_{V}$ effective charge defined in Ref. [49]. We can also make use of the $g_{1}$ scheme, where the strong coupling $\alpha_{g_{1}}\left(Q^{2}\right)$ is determined from the Bjorken sum rule. [50] The coupling $\alpha_{g_{1}}\left(Q^{2}\right)$ has the advantage that it is the best-measured effective charge, and it can be used to extrapolate the definition of the effective coupling to large distances. [51] Since $\alpha_{g_{1}}$ has been measured at intermediate energies, it is particularly useful for studying the transition from small to large distances.

We will show [3] how the LF holographic mapping of effective classical gravity in AdS space, modified by a positive-sign dilaton background, can be used to identify an analytically simple color-confining non-perturbative effective coupling $\alpha_{s}^{A d S}\left(Q^{2}\right)$ as a function of the space-like momentum transfer $Q^{2}=-q^{2}$. This coupling incorporates confinement and agrees well with effective charge observables and lattice simulations. It also exhibits an infrared fixed point at small $Q^{2}$ and asymptotic freedom at large $Q^{2}$. However, the fall-off of $\alpha_{s}^{A d S}\left(Q^{2}\right)$ at large $Q^{2}$ is exponential: $\alpha_{s}^{A d S}\left(Q^{2}\right) \sim e^{-Q^{2} / \kappa^{2}}$, rather than the perturbative QCD (pQCD) logarithmic fall-off. We also show in Ref. [3] that a phenomenological extended coupling can be defined which implements the pQCD behavior.

As will be discussed below, the $\beta$-function derived from light-front holography becomes significantly negative in the non-perturbative regime $Q^{2} \sim \kappa^{2}$, where it reaches a minimum, signaling the transition region from the infrared (IR) conformal region, 
characterized by hadronic degrees of freedom, to a pQCD conformal ultraviolet (UV) regime where the relevant degrees of freedom are the quark and gluon constituents. The $\beta$-function is always negative: it vanishes at large $Q^{2}$ consistent with asymptotic freedom, and it vanishes at small $Q^{2}$ consistent with an infrared fixed point. [52, 5]

Let us consider a five-dimensional gauge field $G$ propagating in $\mathrm{AdS}_{5}$ space in presence of a dilaton background $\varphi(z)$ which introduces the energy scale $\kappa$ in the fivedimensional action. At quadratic order in the field strength the action is

$$
S=-\frac{1}{4} \int d^{5} x \sqrt{g} e^{\varphi(z)} \frac{1}{g_{5}^{2}} G^{2},
$$

where the metric determinant of $\mathrm{AdS}_{5}$ is $\sqrt{g}=(R / z)^{5}, \varphi=\kappa^{2} z^{2}$ and the square of the coupling $g_{5}$ has dimensions of length. We can identify the prefactor

$$
g_{5}^{-2}(z)=e^{\varphi(z)} g_{5}^{-2},
$$

in the AdS action (7) as the effective coupling of the theory at the length scale $z$. The coupling $g_{5}(z)$ then incorporates the non-conformal dynamics of confinement. The five-dimensional coupling $g_{5}(z)$ is mapped, modulo a constant, into the Yang-Mills (YM) coupling $g_{Y M}$ of the confining theory in physical space-time using light-front holography. One identifies $z$ with the invariant impact separation variable $\zeta$ which appears in the LF Hamiltonian: $g_{5}(z) \rightarrow g_{Y M}(\zeta)$. Thus

$$
\alpha_{s}^{A d S}(\zeta)=g_{Y M}^{2}(\zeta) / 4 \pi \propto e^{-\kappa^{2} \zeta^{2}} .
$$

In contrast with the 3-dimensional radial coordinates of the non-relativistic Schrödinger theory, the natural light-front variables are the two-dimensional cylindrical coordinates $(\zeta, \phi)$ and the light-cone fraction $x$. The physical coupling measured at the scale $Q$ is the two-dimensional Fourier transform of the LF transverse coupling $\alpha_{s}^{A d S}(\zeta)$ (9). Integration over the azimuthal angle $\phi$ gives the Bessel transform

$$
\alpha_{s}^{A d S}\left(Q^{2}\right) \sim \int_{0}^{\infty} \zeta d \zeta J_{0}(\zeta Q) \alpha_{s}^{A d S}(\zeta),
$$

in the $q^{+}=0$ light-front frame where $Q^{2}=-q^{2}=-\mathbf{q}_{\perp}^{2}>0$ is the square of the space-like four-momentum transferred to the hadronic bound state. Using this ansatz we then have from Eq. (10)

$$
\alpha_{s}^{A d S}\left(Q^{2}\right)=\alpha_{s}^{A d S}(0) e^{-Q^{2} / 4 \kappa^{2}} .
$$

In contrast, the negative dilaton solution $\varphi=-\kappa^{2} z^{2}$ leads to an integral which diverges at large $\zeta$. We identify $\alpha_{s}^{A d S}\left(Q^{2}\right)$ with the physical QCD running coupling in its nonperturbative domain.

The flow equation (8) from the scale dependent measure for the gauge fields can be understood as a consequence of field-strength renormalization. In physical QCD 
we can rescale the non-Abelian gluon field $A^{\mu} \rightarrow \lambda A^{\mu}$ and field strength $G^{\mu \nu} \rightarrow \lambda G^{\mu \nu}$ in the QCD Lagrangian density $\mathcal{L}_{\mathrm{QCD}}$ by a compensating rescaling of the coupling strength $g \rightarrow \lambda^{-1} g$. The renormalization of the coupling $g_{\text {phys }}=Z_{3}^{1 / 2} g_{0}$, where $g_{0}$ is the bare coupling in the Lagrangian in the UV-regulated theory, is thus equivalent to the renormalization of the vector potential and field strength: $A_{r e n}^{\mu}=Z_{3}^{-1 / 2} A_{0}^{\mu}, G_{r e n}^{\mu \nu}=$ $Z_{3}^{-1 / 2} G_{0}^{\mu \nu}$ with a rescaled Lagrangian density $\mathcal{L}_{\mathrm{QCD}}^{r e n}=Z_{3}^{-1} \mathcal{L}_{\mathrm{QCD}}^{0}=\left(g_{p h y s} / g_{0}\right)^{-2} \mathcal{L}_{0}$. In lattice gauge theory, the lattice spacing $a$ serves as the UV regulator, and the renormalized QCD coupling is determined from the normalization of the gluon field strength as it appears in the gluon propagator. The inverse of the lattice size $L$ sets the mass scale of the resulting running coupling. As is the case in lattice gauge theory, color confinement in AdS/QCD reflects nonperturbative dynamics at large distances. The QCD couplings defined from lattice gauge theory and the soft wall holographic model are thus similar in concept, and both schemes are expected to have similar properties in the nonperturbative domain, up to a rescaling of their respective momentum scales.

\section{Comparison of the Holographic Coupling with Other Effective Charges}

The effective coupling $\alpha^{A d S}\left(Q^{2}\right)$ (solid line) is compared in Fig. 5 with experimental and lattice data. For this comparison to be meaningful, we have to impose the same normalization on the AdS coupling as the $g_{1}$ coupling. This defines $\alpha_{s}^{A d S}$ normalized to the $g_{1}$ scheme: $\alpha_{g_{1}}^{A d S}\left(Q^{2}=0\right)=\pi$. Details on the comparison with other effective charges are given in Ref. [53].

The couplings in Fig. 5(a) agree well in the strong coupling regime up to $Q \sim 1 \mathrm{GeV}$. The value $\kappa=0.54 \mathrm{GeV}$ is determined from the vector meson Regge trajectory. [8]. The lattice results shown in Fig. 5 from Ref. [54] have been scaled to match the perturbative UV domain. The effective charge $\alpha_{g_{1}}$ has been determined in Ref. [53] from several experiments. Fig. 5 also displays other couplings from different observables as well as $\alpha_{g_{1}}$ which is computed from the Bjorken sum rule [50] over a large range of momentum transfer (cyan band). At $Q^{2}=0$ one has the constraint on the slope of $\alpha_{g_{1}}$ from the Gerasimov-Drell-Hearn (GDH) sum rule [55] which is also shown in the figure. The results show no sign of a phase transition, cusp, or other non-analytical behavior, a fact which allows us to extend the functional dependence of the coupling to large distances. As discussed below, the smooth behavior of the AdS strong coupling also allows us to extrapolate its form to the perturbative domain.

The hadronic model obtained from the dilaton-modified AdS space provides a semiclassical first approximation to QCD. Color confinement is introduced by the harmonic oscillator potential, but effects from gluon creation and absorption are not included in this effective theory. The nonperturbative confining effects vanish exponentially at large momentum transfer (Eq. (11)), and thus the logarithmic fall-off from pQCD quantum loops will dominate in this regime. 

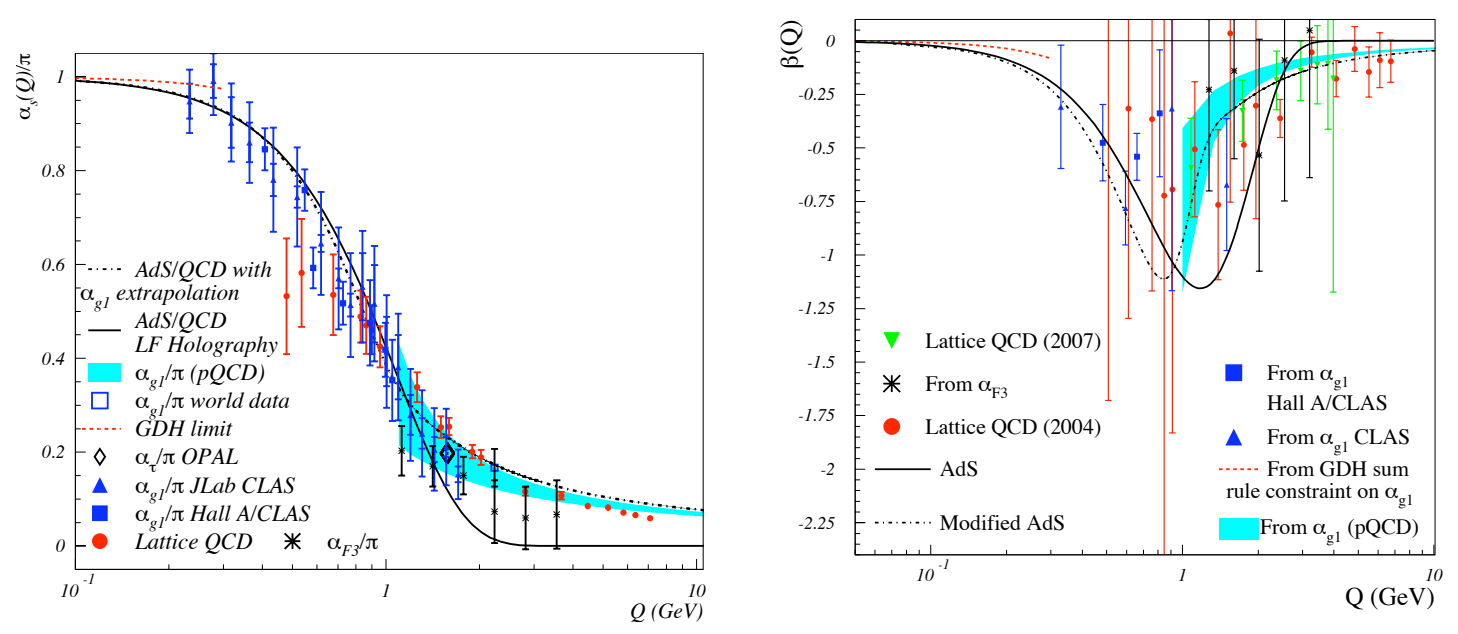

Figure 5: (a) Effective coupling from LF holography for $\kappa=0.54 \mathrm{GeV}$ compared with effective QCD couplings extracted from different observables and lattice results. (b) Prediction for the $\beta$ function compared to lattice simulations, JLab and CCFR results for the Bjorken sum rule effective charge.

The running coupling $\alpha_{s}^{A d S}$ given by Eq. (11) is obtained from a color-confining potential. Since the strong coupling is an analytical function of the momentum transfer at all scales, we can extend the range of applicability of $\alpha_{s}^{A d S}$ by matching to a perturbative coupling at the transition scale, $Q \sim 1 \mathrm{GeV}$, where pQCD contributions become important. In order to have a fully analytical model, we write

$$
\alpha_{M o d i f i e d, g_{1}}^{\text {AdS }}\left(Q^{2}\right)=\alpha_{g_{1}}^{A d S}\left(Q^{2}\right) g_{+}\left(Q^{2}\right)+\alpha_{g_{1}}^{f i t}\left(Q^{2}\right) g_{-}\left(Q^{2}\right)
$$

where $g_{ \pm}\left(Q^{2}\right)=1 /\left(1+e^{ \pm\left(Q^{2}-Q_{0}^{2}\right) / \tau^{2}}\right)$ are smeared step functions which match the two regimes. The parameter $\tau$ represents the width of the transition region. Here $\alpha_{g_{1}}^{A d S}$ is given by Eq. $(11)$ with the normalization $\alpha_{g_{1}}^{A d S}(0)=\pi$ - the plain black line in Fig. 5 and $\alpha_{g_{1}}^{\text {fit }}$ in Eq. (12) is the analytical fit to the measured coupling $\alpha_{g_{1}}$. [53] The couplings are chosen to have the same normalization at $Q^{2}=0$. The smoothly extrapolated result (dot-dashed line) for $\alpha_{s}$ is also shown on Fig. 5. We use the parameters $Q_{0}^{2}=0.8 \mathrm{GeV}^{2}$ and $\tau^{2}=0.3 \mathrm{GeV}^{2}$.

The $\beta$-function for the nonperturbative effective coupling obtained from the LF holographic mapping in a positive dilaton modified AdS background is

$$
\beta^{A d S}\left(Q^{2}\right)=\frac{d}{d \log Q^{2}} \alpha^{A d S}\left(Q^{2}\right)=\frac{\pi Q^{2}}{4 \kappa^{2}} e^{-Q^{2} /\left(4 \kappa^{2}\right)} .
$$

The solid line in Fig. 5 (b) corresponds to the light-front holographic result Eq. (13). Near $Q_{0} \simeq 2 \kappa \simeq 1 \mathrm{GeV}$, we can interpret the results as a transition from the nonperturbative IR domain to the quark and gluon degrees of freedom in the perturbative 
UV regime. The transition momentum scale $Q_{0}$ is compatible with the momentum transfer for the onset of scaling behavior in exclusive reactions where quark counting rules are observed. [56] For example, in deuteron photo-disintegration the onset of scaling corresponds to momentum transfer of $1.0 \mathrm{GeV}$ to the nucleon involved. [57] Dimensional counting is built into the AdS/QCD soft and hard wall models since the AdS amplitudes $\Phi(z)$ are governed by their twist scaling behavior $z^{\tau}$ at short distances, $z \rightarrow 0$. [6]

Also shown on Fig. 5 (b) are the $\beta$-functions obtained from phenomenology and lattice calculations. For clarity, we present only the LF holographic predictions, the lattice results from, [54] and the experimental data supplemented by the relevant sum rules. The width of the aqua band is computed from the uncertainty of $\alpha_{g_{1}}$ in the perturbative regime. The dot-dashed curve corresponds to the extrapolated approximation given by Eq. 12. Only the point-to-point uncorrelated uncertainties of the JLab data are used to estimate the uncertainties, since a systematic shift cancels in the derivative. Nevertheless, the uncertainties are still large. The $\beta$-function extracted from LF holography, as well as the forms obtained from the works of Cornwall [52], Bloch, Fisher et al., [58] Burkert and Ioffe [59] and Furui and Nakajima, [54] are seen to have a similar shape and magnitude.

Judging from these results, we infer that the actual $\beta$-function of QCD will extrapolate between the non-perturbative results for $Q<1 \mathrm{GeV}$ and the pQCD results for $Q>1 \mathrm{GeV}$. We also observe that the general conditions

$$
\begin{gathered}
\beta(Q \rightarrow 0)=\beta(Q \rightarrow \infty)=0, \\
\beta(Q)<0, \text { for } Q>0, \\
\left.\frac{d \beta}{d Q}\right|_{Q=Q_{0}}=0, \\
\frac{d \beta}{d Q}<0, \text { for } Q<Q_{0}, \quad \frac{d \beta}{d Q}>0, \text { for } Q>Q_{0} .
\end{gathered}
$$

are satisfied by our model $\beta$-function obtained from LF holography.

Eq. (14) expresses the fact that QCD approaches a conformal theory in both the far ultraviolet and deep infrared regions. In the semiclassical approximation to QCD without particle creation or absorption, the $\beta$-function is zero and the approximate theory is scale invariant in the limit of massless quarks. 60] When quantum corrections are included, the conformal behavior is preserved at very large $Q$ because of asymptotic freedom and near $Q \rightarrow 0$ because the theory develops a fixed point. An infrared fixed point is in fact a natural consequence of color confinement: [52] since the propagators of the colored fields have a maximum wavelength, all loop integrals in the computation of the gluon self-energy decouple at $Q^{2} \rightarrow 0$. [5] Condition (15) for $Q^{2}$ large, expresses the basic anti-screening behavior of $\mathrm{QCD}$ where the strong coupling vanishes. The $\beta$-function in QCD is essentially negative, thus the coupling increases monotonically from the UV to the IR where it reaches its maximum value: it has a finite value for a theory with a mass gap. Equation (16) defines the transition region at $Q_{0}$ where the beta function has a minimum. Since there is only one hadronic-partonic transition, 
the minimum is an absolute minimum; thus the additional conditions expressed in Eq (17) follow immediately from Eqs. 14, 16). The conditions given by Eqs. (14, 17) describe the essential behavior of the full $\beta$-function for an effective QCD coupling whose scheme/definition is similar to that of the $V$-scheme.

\section{Conclusions}

As we have shown, the combination of Anti-de Sitter space (AdS) methods with lightfront (LF) holography provides a remarkably accurate first approximation for the spectra and wavefunctions of meson and baryon light-quark bound states. We obtain a connection between a semiclassical first approximation to QCD, quantized on the light-front, with hadronic modes propagating on a fixed AdS background. The resulting bound-state Hamiltonian equation of motion in QCD leads to relativistic light-front wave equations in the invariant impact variable $\zeta$ which measures the separation of the quark and gluonic constituents within the hadron at equal light-front time. This corresponds to the effective single-variable relativistic Schrödinger-like equation in the AdS fifth dimension coordinate $z$, Eq. (1). The eigenvalues give the hadronic spectrum, and the eigenmodes represent the probability distributions of the hadronic constituents at a given scale. As we have shown, the light-front holographic mapping of effective classical gravity in AdS space, modified by a positive-sign dilaton background, provides a very good description of the spectrum and form factors of light mesons and baryons.

There are many phenomenological applications where detailed knowledge of the QCD coupling and the renormalized gluon propagator at relatively soft momentum transfer are essential. This includes the rescattering (final-state and initial-state interactions) which create the leading-twist Sivers single-spin correlations in semi-inclusive deep inelastic scattering, [61, 62] the Boer-Mulders functions which lead to anomalous $\cos 2 \phi$ contributions to the lepton pair angular distribution in the unpolarized Drell-Yan reaction, 63] and the Sommerfeld-Sakharov-Schwinger correction to heavy quark production at threshold. [64] The confining AdS/QCD coupling from light-front holography can lead to a quantitative understanding of this factorization-breaking physics. 65.

We have also shown that the light-front holographic mapping of effective classical gravity in AdS space, modified by the same positive-sign dilaton background predicts the form of a non-perturbative effective coupling $\alpha_{s}^{A d S}(Q)$ and its $\beta$-function. The AdS/QCD running coupling is in very good agreement with the effective coupling $\alpha_{g_{1}}$ extracted from the Bjorken sum rule. Surprisingly, the Furui and Nakajima lattice results [54] also agree better overall with the $g_{1}$ scheme rather than the $V$ scheme. Our analysis indicates that light-front holography captures the essential dynamics of confinement. The holographic $\beta$-function displays a transition from nonperturbative to perturbative regimes at a momentum scale $Q \sim 1 \mathrm{GeV}$. It appears to captures the essential characteristics of the full $\beta$-function of QCD, thus giving further support to the application of the gauge/gravity duality to the confining dynamics of strongly coupled QCD. 


\section{Acknowledgments}

Presented by SJB at SCGT09, 2009 International Workshop on Strong Coupling Gauge Theories in the LHC Era, Nagoya, December 8-11, 2009. We thank Volker Burkert, John Cornwall, Sadataka Furui, Philipp Hägler, Wolfgang Korsch, G. Peter Lepage, Takemichi Okui, Joannis Papavassiliou and Anatoly Radyushkin for helpful comments. This research was supported by the Department of Energy contracts DE-AC0276SF00515 and DE-AC05-84ER40150.

\section{References}

[1] J. M. Maldacena, Adv. Theor. Math. Phys. 2, 231 (1998) [Int. J. Theor. Phys. 38, 1113 (1999)] arXiv:hep-th/9711200].

[2] S. S. Gubser, I. R. Klebanov and A. M. Polyakov, Phys. Lett. B 428, 105 (1998) arXiv:hep-th/9802109]; E. Witten, Adv. Theor. Math. Phys. 2, 253 (1998) |arXiv:hep-th/9802150|.

[3] S. J. Brodsky, G. F. de Teramond and A Deur, arXiv:1002.3948 [hep-ph].

[4] A. Deur, V. Burkert, J. P. Chen and W. Korsch, Phys. Lett. B 665, 349 (2008) arXiv:0803.4119 [hep-ph]].

[5] S. J. Brodsky and R. Shrock, Phys. Lett. B 666, 95 (2008) arXiv:0806.1535 [hepth]].

[6] J. Polchinski and M. J. Strassler, Phys. Rev. Lett. 88, 031601 (2002) arXiv:hepth/0109174.

[7] A. Karch, E. Katz, D. T. Son and M. A. Stephanov, Phys. Rev. D 74, 015005 (2006) arXiv:hep-ph/0602229].

[8] G. F. de Teramond and S. J. Brodsky, arXiv:0909.3900 [hep-ph].

[9] O. Andreev and V. I. Zakharov, Phys. Rev. D 74, 025023 (2006) arXiv:hepph/0604204.

[10] F. Zuo, arXiv:0909.4240 [hep-ph].

[11] S. S. Afonin, arXiv:1001.3105 [hep-ph].

[12] S. D. Glazek and M. Schaden, Phys. Lett. B 198, 42 (1987).

[13] P. Hoyer, arXiv:0909.3045 [hep-ph].

[14] P. A. M. Dirac, Rev. Mod. Phys. 21, 392 (1949). 
[15] S. J. Brodsky, H. C. Pauli and S. S. Pinsky, Phys. Rept. 301, 299 (1998) arXiv:hep-ph/9705477.

[16] S. J. Brodsky and G. F. de Teramond, Phys. Rev. Lett. 96, 201601 (2006) arXiv:hep-ph/0602252];

[17] S. J. Brodsky and G. F. de Teramond, Phys. Rev. D 77, 056007 (2008) arXiv:0707.3859 [hep-ph]].

[18] S. J. Brodsky and G. F. de Teramond, Phys. Rev. D 78, 025032 (2008) arXiv:0804.0452 [hep-ph]].

[19] G. F. de Teramond and S. J. Brodsky, Phys. Rev. Lett. 102, 081601 (2009) arXiv:0809.4899 [hep-ph]].

[20] G. F. de Teramond and S. J. Brodsky, arXiv:1001.5193 [hep-ph].

[21] J. Polchinski and M. J. Strassler, JHEP 0305, 012 (2003) arXiv:hep-th/0209211.

[22] Z. Abidin and C. E. Carlson, Phys. Rev. D 77, 095007 (2008) arXiv:0801.3839 [hep-ph]].

[23] C. Amsler et al. (Particle Data Group), Phys. Lett. B 667, 1, (2008).

[24] N. J. Craig and D. Green, JHEP 0909, 113 (2009) arXiv:0905.4088 [hep-ph]].

[25] S. J. Brodsky and G. F. de Teramond, Phys. Lett. B 582, 211 (2004) arXiv:hepth/0310227.

[26] J. Erlich, E. Katz, D. T. Son and M. A. Stephanov, Phys. Rev. Lett. 95, 261602 (2005) arXiv:hep-ph/0501128.

[27] L. Da Rold and A. Pomarol, Nucl. Phys. B 721, 79 (2005) arXiv:hep-ph/0501218.

[28] E. Klempt and A. Zaitsev, Phys. Rept. 454, 1 (2007) arXiv:0708.4016 [hep-ph]].

[29] H. Boschi-Filho, N. R. F. Braga and H. L. Carrion, Phys. Rev. D 73, 047901 (2006) arXiv:hep-th/0507063.

[30] N. Evans and A. Tedder, Phys. Lett. B 642, 546 (2006) arXiv:hep-ph/0609112.

[31] D. K. Hong, T. Inami and H. U. Yee, Phys. Lett. B 646, 165 (2007) arXiv:hepph/0609270.

[32] P. Colangelo, F. De Fazio, F. Jugeau and S. Nicotri, Phys. Lett. B 652, 73 (2007) arXiv:hep-ph/0703316.

[33] H. Forkel, Phys. Rev. D 78, 025001 (2008) arXiv:0711.1179 [hep-ph]]. 
[34] A. Vega and I. Schmidt, Phys. Rev. D 78 (2008) 017703 arXiv:0806.2267 [hep$\mathrm{ph}]$.

[35] K. Nawa, H. Suganuma and T. Kojo, Mod. Phys. Lett. A 23, 2364 (2008) arXiv:0806.3040 [hep-th]].

[36] W. de Paula, T. Frederico, H. Forkel and M. Beyer, Phys. Rev. D 79, 075019 (2009) [arXiv:0806.3830 [hep-ph]].

[37] P. Colangelo, F. De Fazio, F. Giannuzzi, F. Jugeau and S. Nicotri, Phys. Rev. D 78 (2008) 055009 [arXiv:0807.1054 [hep-ph]].

[38] H. Forkel and E. Klempt, Phys. Lett. B 679, 77 (2009) [arXiv:0810.2959 [hep-ph]].

[39] H. C. Ahn, D. K. Hong, C. Park and S. Siwach, Phys. Rev. D 80, 054001 (2009) arXiv:0904.3731 [hep-ph]].

[40] Y. Q. Sui, Y. L. Wu, Z. F. Xie and Y. B. Yang, arXiv:0909.3887 [hep-ph].

[41] H. J. Kwee and R. F. Lebed, JHEP 0801, 027 (2008) arXiv:0708.4054 [hep-ph]].

[42] H. R. Grigoryan and A. V. Radyushkin, Phys. Rev. D 76 (2007) 115007 arXiv:0709.0500 [hep-ph]]; Phys. Rev. D 78 (2008) 115008 arXiv:0808.1243 [hep$\mathrm{ph}]$.

[43] S. J. Brodsky and G. F. de Teramond, arXiv:0802.0514 [hep-ph].

[44] G. F. de Teramond and S. J. Brodsky, Phys. Rev. Lett. 94, 201601 (2005) arXiv:hep-th/0501022].

[45] H. R. Grigoryan and A. V. Radyushkin, Phys. Rev. D 76 (2007) 095007 arXiv:0706.1543 [hep-ph]].

[46] The data compilation is from M. Diehl, Nucl. Phys. Proc. Suppl. 161, 49 (2006) arXiv:hep-ph/0510221].

[47] G. Grunberg, Phys. Lett. B 95, 70 (1980); Phys. Rev. D 29, 2315 (1984); Phys. Rev. D 40, 680 (1989).

[48] S. J. Brodsky and H. J. Lu, Phys. Rev. D 51, 3652 (1995) arXiv:hep-ph/9405218; S. J. Brodsky, G. T. Gabadadze, A. L. Kataev and H. J. Lu, Phys. Lett. B 372, 133 (1996) arXiv:hep-ph/9512367.

[49] T. Appelquist, M. Dine and I. J. Muzinich, Phys. Lett. B 69, 231 (1977).

[50] J. D. Bjorken, Phys. Rev. 148, 1467 (1966).

[51] A. Deur, arXiv:0907.3385 [nucl-ex]. 
[52] J. M. Cornwall, Phys. Rev. D 26, 1453 (1982).

[53] A. Deur, V. Burkert, J. P. Chen and W. Korsch, Phys. Lett. B 650, 244 (2007) arXiv:hep-ph/0509113].

[54] S. Furui and H. Nakajima, Phys. Rev. D 70, 094504 (2004); S. Furui, arXiv:0908.2768 [hep-lat].

[55] S. D. Drell and A. C. Hearn, Phys. Rev. Lett. 16, 908 (1966); S. B. Gerasimov, Sov. J. Nucl. Phys. 2 (1966) 430 [Yad. Fiz. 2 (1966) 598].

[56] S. J. Brodsky and G. R. Farrar, Phys. Rev. Lett. 31, 1153 (1973).

[57] H. Gao and L. Zhu, AIP Conf. Proc. 747, 179 (2005) [arXiv:nucl-ex/0411014.

[58] J. C. R. Bloch, Phys. Rev. D 66, 034032 (2002) [arXiv:hep-ph/0202073]; P. Maris and P. C. Tandy, Phys. Rev. C 60, 055214 (1999) |arXiv:nucl-th/9905056|; C. S. Fischer and R. Alkofer, Phys. Lett. B 536, 177 (2002) |arXiv:hepph/0202202]; C. S. Fischer, R. Alkofer and H. Reinhardt, Phys. Rev. D 65, 094008 (2002) arXiv:hep-ph/0202195]; R. Alkofer, C. S. Fischer and L. von Smekal, Acta Phys. Slov. 52, 191 (2002) arXiv:hep-ph/0205125]; M. S. Bhagwat, M. A. Pichowsky, C. D. Roberts and P. C. Tandy, Phys. Rev. C 68, 015203 (2003) arXiv:nucl-th/0304003].

[59] V. D. Burkert and B. L. Ioffe, Phys. Lett. B 296, 223 (1992); J. Exp. Theor. Phys. 78, 619 (1994) [Zh. Eksp. Teor. Fiz. 105, 1153 (1994)].

[60] G. Parisi, Phys. Lett. B 39, 643 (1972).

[61] S. J. Brodsky, D. S. Hwang and I. Schmidt, Phys. Lett. B 530, 99 (2002) arXiv:hep-ph/0201296].

[62] J. C. Collins, Phys. Lett. B 536, 43 (2002) arXiv:hep-ph/0204004.

[63] D. Boer, S. J. Brodsky and D. S. Hwang, Phys. Rev. D 67, 054003 (2003) arXiv:hep-ph/0211110].

[64] S. J. Brodsky, A. H. Hoang, J. H. Kuhn and T. Teubner, Phys. Lett. B 359, 355 (1995) arXiv:hep-ph/9508274.

[65] J. Collins and J. W. Qiu, Phys. Rev. D 75, 114014 (2007) arXiv:0705.2141 [hep$\mathrm{ph}]$. 